\title{
Effects of MDA, CS intensity, and UCS intensity on the rabbit's conditioned nictitating membrane response
}

\author{
KIMBERLY K. KIRKPATRICK-STEGER, SUSAN VANDER LINDEN, and I. GORMEZANO \\ University of Iowa, Iowa City, Iowa
}

\begin{abstract}
The first experiment in a previous series of investigations in which we were concerned with assessing the dose effects of (+)-3,4-methylenedioxyamphetamine (MDA) on classical conditioning of the rabbit's nictitating membrane response revealed an enhancement of conditioning to a light conditioned stimulus (CS) and an impairment in conditioning to a tone CS. A second study revealed that MDA's effects on conditioning could not be attributed to the drug's effects on nonassociative contributors to response occurrence. Accordingly, in several subsequent investigations we sought to localize MDA's conditioning effects in the organism's processing of the CS. These studies revealed that MDA attenuated tone-CS intensity and the tone CS's unconditioned excitatory properties. The final study in the series revealed that MDA enhanced the frequency of unconditioned response (UCRs) across a range of unconditioned stimulus (UCS) intensities. These simple effects of MDA on the processing of the CS and UCS/UCR would, on the basis of empirical and theoretical considerations, account for the drug's effect on conditioning. As yet, however, an assessment of the possible interactive effects of MDA's CS and UCS/UCR effects on conditioning has not been made. Accordingly, to provide one means for assessing whether or not MDA interacted with CS and UCS intensity, in the present study we employed a between-groups MDA dosage manipulation and withingroup CS and UCS intensity manipulation. Consistent with theoretical expectations was a pattern of responding that yielded a robust MDA dosage $\times$ CS intensity $\times$ UCS intensity interaction.
\end{abstract}

Our purpose in the present study was to determine the effects of (+)-3,4-methylenedioxyamphetamine (MDA) dosage $(0,2$, and $4 \mathrm{mg} / \mathrm{kg})$ and conditioned stimulus (CS; 55,70 , and $85 \mathrm{~dB}$ ) and unconditioned stimulus (UCS; .75, 2.0 , and $4.0 \mathrm{~mA}$ ) intensity on the classically conditioned rabbit nictitating membrane response (NMR).

Over the last decade, employing the rabbit NMR preparation, we have conducted an extensive series of investigations on the effects of drugs (e.g., haloperidol, scopolamine, morphine, and d-lysergic acid diethylamide) on learning. In these studies, the NMR preparation has permitted us to design experiments for distinguishing between drug effects on three postulated components of the conditioning process (Gormezano, Kehoe, \& Marshall, 1983): (1) sensory processing of stimuli; (2) associative learning; and (3) motor functioning. Specifically, we have developed a battery of experimental procedures that have permitted us to isolate a drug's effect on one or more of these components of the conditioning process. Recently, we have employed this battery of procedures to assess the effect of MDA on the postulated components of the con-

This research was supported by National Institute on Drug Abuse Grant DAO-5075-02 and The University of Iowa Foundation (through the generous contributions of D. Boatman, H. Gormezano, S. L. Nusser, and W. G. Nusser). The authors extend their thanks to J. Jensen for her assistance in preparation of the manuscript. Correspondence concerning this manuscript should be sent to I. Gormezano, Department of Psychology, University of lowa, Iowa City, IA 52242. ditioning process (Kirkpatrick-Steger, Vander Linden, \& Gormezano, 1991). The first study, in a series of five investigations, carried out to assess MDA's dose effect on classical conditioning of the rabbit's NMR, revealed an enhancement of conditioning to a light CS and an impairment in conditioning to a tone CS. A second experiment revealed that MDA's effects on NMR conditioning could not be attributed to the drug's effects on nonassociative contributors to response occurrence. Accordingly, in several subsequent investigations we sought to localize MDA's conditioning effects in the organism's processing of the CS. These studies revealed that MDA attenuated tone-CS intensity and the tone CS's unconditioned excitatory properties. The final study in the series revealed that MDA enhanced the frequency of unconditioned responses (UCRs) across a range of UCS intensities. These simple effects of MDA on the processing of the CS and UCS/UCR would, on the basis of empirical and theoretical considerations, account for the drug's effect on conditioning. As yet, however, an assessment of the possible interactive effects on MDA's CS and UCS/UCR effects on conditioning has not been made. Yet theoretical accounts of conditioning lead one to expect that they would interact to affect the conditioning process. Accordingly, to provide one means for assessing MDA's interactions with CS intensity and UCS intensity and their effects on NMR conditioning, in the present study we employed a betweengroups MDA dosage manipulation and within-group CS and UCS intensity manipulations. 


\section{METHOD}

\section{Subjects}

The subjects were 42 naive male and female (New Zealand white albino) rabbits, each 80-100 days old and weighing approximately $2 \mathrm{~kg}$ on arrival from Knapp Creek Rabbitry (Amana, Iowa).

\section{Apparatus}

Briefly, the transducer for recording the NMR consisted of a phototransistor assembly directly coupled to a nylon loop sutured in the NM of the right eye (Gormezano \& Gibbs, 1988). The transducer converted NM movements to analog electrical signals subjected to an analog-to-digital (A/D) conversion at a 5-msec sampling rate and resolution of $0.06 \mathrm{~mm}$ of actual membrane movement by an Apple II/FIRST hardware-software system (Scandrett \& Gormezano, 1980). The conditioning chambers were fabricated from fireproof filing cabinets, to which individual blowers, exhausts, and stimulus panels had been added. Three CSs were employed. An 800 -msec, $1000-\mathrm{Hz}$ tone of 55,70 , or $85 \mathrm{~dB}$ (SPL) was delivered through an 11.4-cm speaker mounted in the center of the stimulus panel; two 6-W, 24-V dc houselights were mounted on each side of the speaker; and the UCS was a $100-\mathrm{msec}, 60-\mathrm{Hz}$ shock of $.75,2.0$, or $4.0 \mathrm{~mA}$ delivered to wound-clip electrodes implanted $10 \mathrm{~mm}$ apart and $15 \mathrm{~mm}$ posterior to the dorsal canthus of the rabbit's right eye.

\section{Procedure}

All animals received 1 day of preparation, 1 day of adaptation, and 6 days of acquisition training. On the preparation day, a loop of surgical nylon was sutured into the rabbit's right NM and the surrounding hair was removed. On the adaptation day, UCS electrodes (Autoclip wound clips) were applied. The animals were then secured in a Plexiglas restrainer and placed in the conditioning chambers for a period equal to the length of the subsequent acquisition sessions. To obtain an index of the base rate of responding, the session was analyzed for responses occurring at times corresponding to the CS-UCS observation interval of the subsequent acquisition sessions. Following adaptation, subjects were randomly assigned to one of three MDA dose conditions of 0 $(n=13), 2(n=15)$, and $4 \mathrm{mg} / \mathrm{kg}(n=14)$ for 6 days of acquisition training, with each session consisting of 81 CS-UCS pairings at intertrial intervals of 50,60 , and $70 \mathrm{sec}$ (mean of $60 \mathrm{sec}$ ). The 81 trials were composed of nine 9-trial blocks obtained from the factorial combination of 3 tone-CS $(55,70$, and $85 \mathrm{~dB})$ and 3 shock-UCS $(.75,2.0$, and $4.0 \mathrm{~mA}$ ) intensities presented in a randomized sequence for each of the nine 9-trial blocks within and across days. Throughout the experiment, a conditioned response (CR) was defined as any NMR extension exceeding $0.5 \mathrm{~mm}$ and occurring within the CS-UCS interval.

\section{Drugs}

MDA [(+)-3,4-methylenedioxyamphetamine] obtained from the National Institute on Drug Abuse (Bethesda, MD) was dissolved in sterile, nonpyrogenic $0.9 \%$ sodium chloride solution. Approximately $30 \mathrm{~min}$ before each experimental session, the vehicle (0) or MDA ( 2 and $4 \mathrm{mg} / \mathrm{kg}$ ) was injected into the marginal ear vein of each animal via a Harvard infusion pump (Model 975) in a volume of $0.4 \mathrm{ml} / \mathrm{kg}$ at a rate of $3 \mathrm{ml} / \mathrm{min}$.

\section{RESULTS}

The frequency, latency, and amplitude of CRs were obtained throughout the 6 days of CS-UCS training. The data for each measure were plotted, and an analysis of variance was performed. An examination of the plots (figures not shown) and the statistical analyses revealed that the most robust and systematic effects were obtained for CR amplitude; our presentation of results will be restricted to this dependent measure accordingly.

The most salient findings of the study are portrayed in Figures 1 and 2. The panels of Figure 1 display the simple effects of CS intensity (left panel) and UCS intensity (middle panel), and the interactive effects of CS intensity and UCS intensity (right panel) on CR amplitude. An examination of these panels reveals that CR amplitude was a monotonic increasing function of both CS and UCS intensity and that, as clearly seen in the right panel, CS and UCS intensity interacted to produce effects on CR amplitude. Specifically, as UCS intensity increased, the effects of increasing CS intensity on CR amplitude were attenuated.

The panels of Figure 2 portray the combined effects of MDA dosage, CS intensity, and UCS intensity on CR amplitude. An examination of the left panel of the figure reveals that the CS-intensity-MDA dosage relationship yielded a concave-down function. Moreover, with an increase in UCS intensity from .75 to $4.0 \mathrm{~mA}$, displayed across the panels from left to right, there was an increase
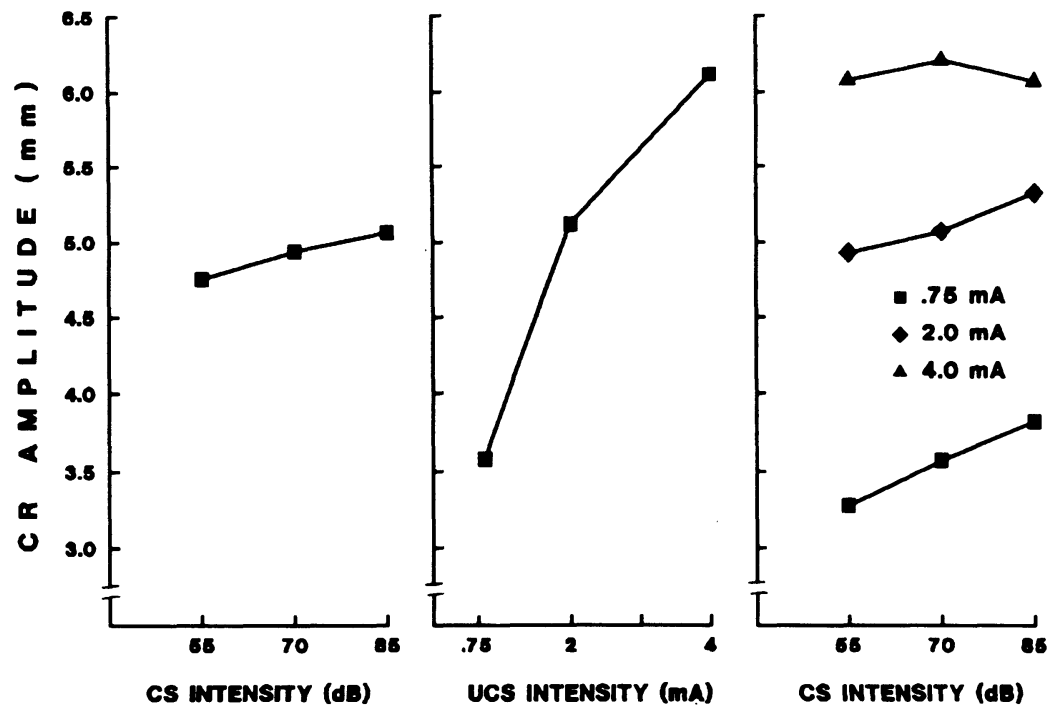

Figure 1. The effects of conditioned stimulus (CS) intensity (left panel), unconditioned stimulus (UCS) intensity (middle panel), and CS intensities at varying UCS intensities (right panel) on conditioned response (CR) amplitude. 


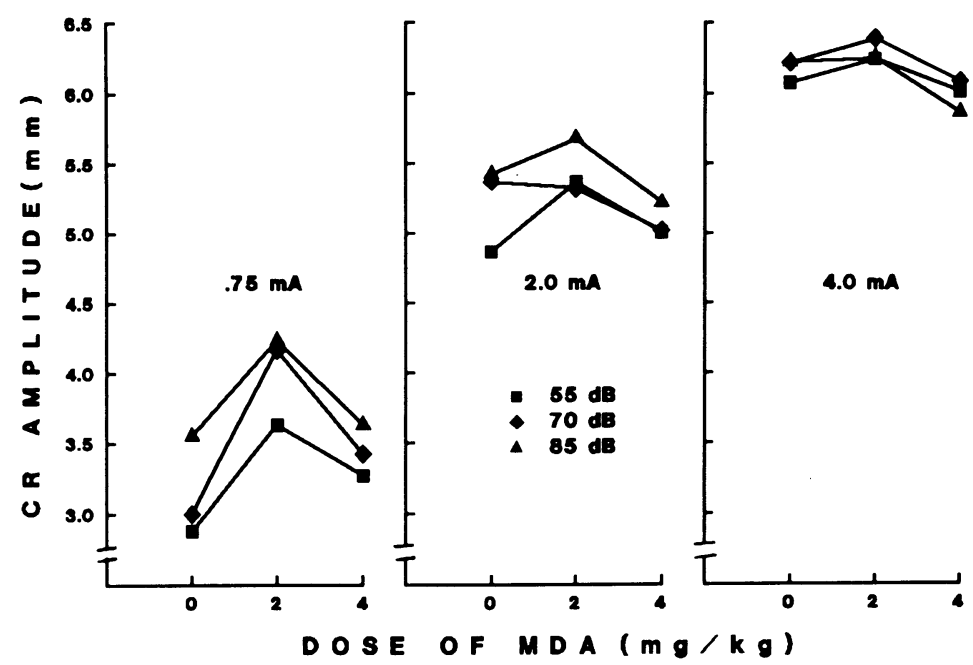

Figure 2. The effects of MDA dose and conditioned stimulus (CS) intensity at unconditioned stimulus (UCS) intensities of $.75 \mathrm{~mA}$ (left panel), $2.0 \mathrm{~mA}$ (middle panel), and $4.0 \mathrm{~mA}$ (right panel) on conditioned response (CR) amplitude.

in CR amplitude at all CS intensities but a gradual flattening in these functions with increased MDA dosage. In particular, MDA substantially decremented $C R$ amplitude at the 4-mA UCS intensity, an effect that clearly cannot be attributed to a "floor" effect on the measurement of CR amplitude.

The analysis of variance performed on CR amplitude confirmed the descriptive aspects of the data portrayed in Figures 1 and 2 . Specifically, the analysis revealed significant effects (all $p s<.05)$ of CS intensity $[F(2,781)=$ $21.19]$ and UCS intensity $[F(1,78)=86.00]$, and significant interactions of CS intensity $\times$ UCS intensity $[F(4,156)$ $=19.27]$ and CS intensity $\times$ UCS intensity $\times$ MDA dosage $[F(8,156)=6.55]$. The analysis also revealed a significant source of variation for the nine trial blocks $[F(8,312)=31.26]$, which reflected a modest withinsession decrement in CR amplitude over trial blocks. In addition, trial blocks revealed first- and second-order interactions with the other significant sources of variation. However, the nature of these interactions did not in any respect alter our characterization of the effects of those significant sources of variation.

\section{DISCUSSION}

The principal finding in the present investigation was that with a betweengroups MDA dosage manipulation and within-group CS intensity and UCS intensity manipulations it has been possible to observe main and interactive effects of MDA dosage, CS intensity, and UCS intensity on CR acquisition. Although the main effects of these variables on $\mathrm{CR}$ acquisition are well known (Gormezano \& Moore, 1969), this assessment of their interactive effects in a within-group design is a new observation of potential theoretical interest. Specifically, in extant theoretical accounts of conditioning it has traditionally been assumed that UCS intensity effects operate during trial to affect CR acquisition, whereas CS intensity is assumed to operate both during and after trial to effect CR elicitation. However, in the present investigation, a conditional analysis of trial-by-trial outcome failed to reveal significant conditional effects of CS or UCS intensity on first-, second-, and third-order conditional occurrences of CRs. Although we are not presuming to "prove the null hypothesis," the statistical power of our analyses was considerable-suggesting, perhaps, that the substantial and robust first-order interaction of CS intensity $\times$ UCS intensity may have operated to augment $\mathrm{CR}$ amplitudes until yielding comparable amplitudes across all CS intensities at the 4-mA UCS intensity, thereby masking the trial-by-trial conditional assessments.

Our previous investigations of MDA's effects on conditioning (Kirkpatrick-Steger et al., 1991) revealed a dose-dependent (0-, 1-, 2-, and $4-\mathrm{mg} / \mathrm{kg}$ ) decrementing of both the rate of CR acquisition and overall CR frequency to a tone CS. These effects were localized within MDA's attenuation of CS intensity. On the other hand, MDA was also observed to produce a dose-dependent increase in the frequency of UCRs across a range of UCS intensities. Accordingly, in the present investigation, the between-groups manipulation of MDA dosage might have been expected to express itself reliably as affecting the simple effects of CS intensity and UCS intensity or, at the least, in a first-order interaction of MDA dose $\times$ CS intensity or MDA dose $\times$ UCS intensity. Nevertheless, the significant second-order interaction of MDA dose $\times$ CS intensity $\times$ UCS intensity confirmed the reliability of an observed pattern of responding that was in accord with previous empirical findings and theoretical expectations.

\section{REFERENCES}

Gormezano, I., \& GibBs, C. M. (1988). Transduction of the rabbit's nictitating membrane response. Behavior Research Methods, Instruments, \& Computers, 20, 18-21.

Gormezano, I., Kehoe, E. J., \& Marshall, B. S. (1983). Twenty years of classical conditioning research with the rabbit. In J. M. Sprague \& A. N. Epstein (Eds.), Progress in psychobiology and physiological psychology (Vol. 10, pp. 197-275). New York: Academic Press.

Gormezano, I., \& MOORE, J. W. (1969). Classical conditioning: Empirical relationships. In M. M. Marx (Ed.), Learning: Processes (pp. 133-168). Toronto: Macmillan.

KirkPatrick-Steger, K., Vander Linden, S., \& Gormezano, I. (1991). Effects of MDA on classical conditioning of the rabbit nictitating membrane response. Pharmacology, Biochemistry \& Behavior, 39, 183-189.

SCANDRETT, J., \& GoRmeZANO, I. (1980). Microprocessor control and A/D data acquisition in classical conditioning. Behavior Research Methods \& Instrumentation, 12, 120-125. 